\title{
Age-group targeted testing for COVID-19 as new prevention strategy
}

\section{Ranjit Kumar Upadhyay ( $\nabla$ ranjitupadhyay@iitism.ac.in )}

Indian Institute of Technology(Indian School of Mines) Dhanbad https://orcid.org/0000-0002-12107804

\section{S. Chatterjee}

Indian Institute of Science Education and Research Kolkata, 741246, West Bengal, India.

\section{S. Saha}

Indian Institute of Science Education and Research Kolkata, 741246, West Bengal, India.

\section{R.K. Azad}

Department of Biological Sciences, College of Science, University of North Texas, Denton, Texas 76203, USA.

\section{Research Article}

Keywords: Epidemic model, COVID-19, Age-structured SIQR model, Basic reproductive ratio, Age-targeted testing

Posted Date: May 29th, 2020

DOl: https://doi.org/10.21203/rs.3.rs-31786/v1

License: (c) (1) This work is licensed under a Creative Commons Attribution 4.0 International License. Read Full License

Version of Record: A version of this preprint was published at Nonlinear Dynamics on August 1st, 2020. See the published version at https://doi.org/10.1007/s11071-020-05879-x. 


\title{
Age-group targeted testing for COVID-19 as new prevention strategy
}

\author{
Ranjit Kumar Upadhyay, ${ }^{1 *}$ Sourin Chatterjee, ${ }^{2}$ Satvik Saha ${ }^{2}$, Rajeev K. Azad ${ }^{3,4}$ \\ ${ }^{1}$ Department of Mathematics \& Computing, \\ Indian Institute of Technology (Indian School of Mines) Dhanbad, Jharkhand, India, 826004 \\ ${ }^{2}$ Indian Institute of Science Education and Research Kolkata, \\ West Bengal, India, 741246. \\ ${ }^{3}$ Department of Biological Sciences, \\ College of Science, University of North Texas, Denton, Texas 76203, USA \\ ${ }^{4}$ Department of Mathematics, \\ College of Science, University of North Texas, Denton, Texas 76203, USA \\ ${ }^{*}$ To whom correspondence should be addressed; Tel: +91-9431126485
}

Robust testing and tracing are key to fighting the menace of coronavirus disease 2019 (COVID-19). This outbreak has progressed with a tremendous impact on human life, society and economy. In this paper, we propose an agestructured SIQR model to track the progression of the pandemic in India, Italy and USA, taking into account the different age-structures of these countries. We have made predictions about the disease dynamics, identified the most infected age-groups and analyzed the effectiveness of social distancing measures taken in the early stages of infection. The basic reproductive ratio $R_{0}$ has been numerically calculated for each country. We propose a strategy of age-targeted testing, with increased testing in the most proportionally infected age-groups. We observe a marked flattening of the infection curve upon 


\section{simulating increased testing in the 15-40 year age-groups in India. Thus, we conclude that social distancing and widespread testing are effective methods of control, with emphasis on testing and identifying the hotspots of highly in- fected populations.}

\section{INTRODUCTION}

News of a pneumonia outbreak in Wuhan, China was reported to WHO on 31 December 2019. This disease, called COVID-19 by WHO, was found to be caused by a new strain of coronavirus, SARS-CoV-2. It has now spread to almost every part of the world (1). WHO declared this outbreak a 'Public Health Emergency of International Concern' on 30 January 2020. As of 30 April 2020, it has caused 2,33,824 deaths with 33,03,544 confirmed cases of infection. Till date, USA has the largest number of fatalities, followed by Italy. USA reports 1,095,023 cases, with 3,226 cases per million people, though the statistics have still been evolving due to the large variability in testing performed by different countries as of now (2).

In the absence of any vaccines or drugs to treat the novel coronavirus disease, COVID-19, effective non-pharmaceutical interventions are needed to contain the epidemic and minimize morbidity and mortality associated with this respiratory disease. As the COVID-19 pandemic has swept across the globe, now affecting almost all countries, measures for mitigation have been put in place that include strict lockdown to less restrictive people movement but all aiming to achieve social distancing of different degrees to balance the socioeconomic impacts of the lockdown and the disease. Robust testing and tracing are key to containing the pandemic and effectively flattening the infection curve. Governments and health agencies have banked on mathematical models to guide towards the goal of optimizing available resources to attain maximal benefits of mitigation measures. Mathematical models are often based on certain assumptions, however, these are continually improved upon through adjustments guided by emerging data 
and eventually, these models become more reliable in helping navigate through such situations. Here, we leverage the emerging information from COVID-19 in different countries, mainly USA, Italy, and India, to develop a COVID-19 specific model that can inform on effective interventions for coronavirus containment. As people of different age-groups have responded differently to coronavirus, we utilized the age-stratified data of COVID-19 to develop a system that can inform on more effective prevention strategies. We particularly focused on India where COVID-19 seems to have not peaked yet despite the most restrictive lockdown imposed for over a month now. Our model recommends that testing and tracing be ramped up in the 15-40 year age group population in India in order to flatten the infection curve in shortest time possible in the current situation. We further demonstrate this by computing the basic reproductive ratio $R_{0}$ at different times and following an age-group targeted intervention.

The novel coronavirus is thought to have originated in bats and eventually infected humans, due to the similarity in the genome sequence of SARS-CoV-2 to that of a bat coronavirus (3). Human to human transmission has also been established. So far, we observe different transmission and fatality rates in different countries. One of the main reasons for this is differing agegroups and social contact structures. In order to study this effect, we use social contact matrices, which show contact patterns of an age-group with others and are used to parametrize mathematical methods to understand the transmission patterns. Schenzle (4) used an age-structured SEIR model with 21 age-groups to study the spread of measles, a disease which mostly affects children. This method has previously been applied to respiratory diseases like influenza (5), pertussis (6), and varicella (7). Given that COVID-19 transmission patterns are very similar to that of respiratory diseases caused by other viruses, we can get valuable information by studying COVID-19 disease dynamics through an age-structured model.

In the absence of any vaccine to prevent the disease, the scenarios in China and many other countries have shown that social distancing, isolating infected populations and quarantine are 
effective ways of containing the epidemic. After receiving optimal therapy, a quarter of critically ill patients still die, signifying that the host response to the virus is an important factor (8). Thus, the government and hospitals need to procure supportive care equipments in sufficient amounts. Initiating the process of flattening the curve by these above mentioned methods provides government the time to prepare for supportive care. With social distancing becoming a preventive strategy, many countries have announced partial or complete lockdowns. Numerous companies are also advising their employees to 'work from home'. Due to this, patterns of mixing between people change, the effects of which are hard to represent with classical compartmental disease models. However, it is essential to understand these changing contact patterns in order to more accurately model the disease dynamics. Age-structured models offer better approximations of reality, and also give health organizations better tools to develop age-group targeted control strategies.

Here, we have simulated the spread of novel coronavirus using such an age-structured SIQR model. We have fitted our model to the current situations in Italy and USA, and have estimated age-wise mortality rates. Side by side, we compared this with the scenario in India. We have also analysed the success of lockdown measures adopted by these countries qualitatively, and have projected the effects of further lockdowns. Finally, we have proposed a novel method of age-group targeted testing to tackle the situation and have also showed how it can help in flattening the curve effectively.

\section{MATERIALS AND METHODS}

Mathematical model formulation The mathematical framework of an age-structured SIQR epidemiological model is formulated. In order to construct the model, our assumptions are stated as follows: The entire population, $N$, is divided into four compartments: susceptible population, $S$ (which are under risk of contracting the infection), infected population, $I$, (which 
consists of infectious, both symptomatic and asymptomatic, or untested individuals), quarantined population, $Q$, (which are removed from all contacts within the entire population, and are hence not infectious), and recovered class, $R$ (which are recovered from infection). Therefore, we have $N=S+I+Q+R$. In order to keep track of disease induced death, we assign an additional compartment of fatalities, $F$. Each population of SIQR and $F$ is further subdivided into $M$ age classes. Individuals within the same compartment interact with other individuals proportional to a coefficient of interaction $C_{i j}$, which specifies the average contact between age classes $i$ and $j$, with $0<i, j \leq M$. There is no recruitment of the susceptible population and no natural death in any compartment. There is also no aging of individuals. The disease is transmitted from the infected to the susceptible population with age class $i$, i.e. compartment $S_{i}$, at a rate $\beta \lambda_{i}$. Here, $\beta$ is the transmission probability, and $\lambda_{i}$ is the weighted coefficient of contact of age class $i$ with the entire infected population.

$$
\lambda_{i}=\sum_{j=1}^{M}\left[C_{i j} \frac{I_{i}}{S_{j}+I_{j}+R_{j}}\right]
$$

Individuals from compartment $S_{i}$ move into compartment $I_{i}$ and become infective immediately. Individuals from compartment $I_{i}$ move into compartment $Q_{i}$ at a rate $\delta_{i}$, and are no longer infectious. The infected and quarantined populations, $I_{i}$ and $Q_{i}$, recover and move into the recovered population, $R_{i}$, at rates $\epsilon$ and $\gamma$ respectively. The populations $I_{i}$ and $Q_{i}$ suffer disease induced death, at a common rate of $\mu_{i}$.

A schematic diagram of the interacting population is presented in Fig. 1. Thus the transmission process is formulated by the following system of differential equations:

$$
\begin{aligned}
\frac{d S_{i}}{d t} & =-\beta \lambda_{i} S_{i} \\
\frac{d I_{i}}{d t} & =\beta \lambda_{i} S_{i}-\left(\delta_{i}+\epsilon+\mu_{i}\right) I_{i} \\
\frac{d Q_{i}}{d t} & =\delta_{i} I_{i}-\left(\gamma+\mu_{i}\right) Q_{i}
\end{aligned}
$$




$$
\begin{aligned}
\frac{d R_{i}}{d t} & =\epsilon I_{i}+\gamma Q_{i}, \\
\frac{d F_{i}}{d t} & =\mu_{i}\left(I_{i}+Q_{i}\right) .
\end{aligned}
$$

The initial conditions are $S_{i}(0)>0, I_{i}(0)>0, Q_{i}(0)>0, R_{i}(0)=0, F_{i}(0)=0$. All the parameters in the system are positive quantities.

We break down the age-structured social contact matrix, $C=\left[C_{i j}\right]_{M \times M}$, into the contributions from households, workplaces, schools (all educational institutions) and other areas (market places, restaurants, cinema halls, shopping malls etc.), represented by $C_{H}, C_{W}, C_{S}$ and $C_{O}$ respectively. Each of these is weighted with coefficients $\alpha_{H}, \alpha_{W}, \alpha_{S}$ and $\alpha_{O}$, which we change over time to reflect the effect of lockdown on social contact.

$$
C=\alpha_{H} C_{H}+\alpha_{W} C_{W}+\alpha_{S} C_{S}+\alpha_{O} C_{O}
$$

For example, during the time period when all educational institutes are closed, we set $\alpha_{S}=$ 0. We note that some countries have announced partial lockdowns, in which case the coefficients $\alpha$ have been fitted to existing data. It must also be noted that even during a complete lockdown, the contributions to the contact matrix from work and other areas are never zero, as people involved in essential services continue work and marketplaces must operate to some degree. Lockdowns also induce an increase in household contact, as people are staying at home more (9). For numerical simulation, we have collected data on the times and nature of lockdown imposed, starting from the closure of schools and universities to complete lockdowns (10-12). A complete lockdown in India was declared on 25 March 2020. USA declared a national emergency on 13 March 2020, followed by various statewise guidelines and orders to "stay at home'. Italy had also proceeded towards lockdown step by step, with a lockdown of the northern provinces on 8 March 2020, and a nationwide lockdown from 10 March 2020 onwards.

The parameters $\delta_{i}, \gamma$ and $\epsilon$ have been assumed, using known rates of infection, recovery, and 
the first appearance of symptoms. We assume the onset of symptoms, detection and isolation in 5.2 days on average (13) for infected individuals. We also assume that an individual stays quarantined for 14 days, which is the average recovery period for a symptomatic individual (14). In addition, we assume that infected individuals, either asymptomatic or untested, can proceed straight to recovery after 15 days on average. The parameters $\beta$ and $\mu_{i}$ have been fitted to existing case and fatality data. The values of $\beta$ for India, USA and Italy are 0.024, 0.034 and 0.041 respectively. The values of the remaining parameters have been listed in Tables S2 and S4. It must be noted that we choose to interpret currently reported cases to belong in the quarantined compartment, $Q$, which is a subset of the total infected population. We have also chosen our initial conditions such that $I_{i}(0)=Q_{i}(0)$, on the assumption that cases are underreported where exactly half of all infected individuals are identified (quarantined).

For India and Italy, we use $M=16$ age classes, equally divding the range of ages 0-80 years. Due to unavailability of data, we use $M=15$ age classes for the USA, over a range 0-75 years. For each country, we have set up $5 M$ differential equations, which we have integrated using the python module 'numpy'. We have collected COVID-19 case and mortality data upto 30 April 2020 for each country from Worldometers (2). We have also collected age-structure data from PopulationPyramid (15), and social contact data from Prem et al. (16). The age classes and initial susceptible population data are presented in Table S3. Case and mortality data have been plotted in Fig. 2 against our simulated data.

Calculation of the basic reproductive ratio Here, we find the basic reproduction number $R_{0}$ using the next generation method (17). We first linearise the system of equations (2) at disease free equilibrium, where $I_{i}=Q_{i}=R_{i}=F_{i}=0$. Hence, when the infected and quarantined populations are small, their dynamics are described by the following system.

$$
\dot{I}_{i}=\sum_{j=1}^{M}\left[\beta C_{i j} \frac{N_{i}}{N_{j}} I_{j}\right]-\left(\delta_{i}+\epsilon+\mu_{i}\right) I_{i},
$$




$$
\dot{Q}_{i}=\delta_{i} I_{i}-\left(\gamma+\mu_{i}\right) Q_{i} .
$$

We collect the infected and quarantined compartments in the vector $\vec{v}=\left(I_{1} \cdots I_{M} Q_{1} \cdots Q_{M}\right)^{T}$. We can thus rewrite the system (4) in the form $\frac{d}{d t} \vec{v}=L \vec{v}$, where $L$ is the $2 M \times 2 M$ matrix described as follows.

$$
\begin{aligned}
L & =\left(\begin{array}{ll}
L^{I I} & L^{I Q} \\
L^{Q I} & L^{Q Q}
\end{array}\right), \\
L_{i j}^{I I} & = \begin{cases}\beta C_{i j} \frac{N_{i}}{N_{j}}-\left(\delta_{i}+\epsilon+\mu_{i}\right), & i=j \\
\beta C_{i j} \frac{N_{i}}{N_{j}}, & i \neq j\end{cases} \\
L_{i j}^{I Q} & =0, \\
L_{i j}^{Q I} & = \begin{cases}\delta_{i}, & i=j \\
0, & i \neq j\end{cases} \\
L_{i j}^{Q Q} & = \begin{cases}-\left(\gamma+\mu_{i}\right), & i=j \\
0 . & i \neq j\end{cases}
\end{aligned}
$$

We further break $L=T-V$, where the transmission matrix, $T$, represents the influx of newly infected individuals, and the transition matrix, $V$, represents the movement between the infected compartments. They are calculated numerically as follows.

$$
\begin{aligned}
T & =\left(\begin{array}{ll}
T^{I I} & T^{I Q} \\
T^{Q I} & T^{Q Q}
\end{array}\right), \\
T_{i j}^{I I} & = \begin{cases}\beta C_{i j} \frac{N_{i}}{N_{j}}, & i=j \\
0, & i \neq j\end{cases} \\
T_{i j}^{I Q} & =T_{i j}^{Q I}=T_{i j}^{Q Q}=0, \\
V & =T-L .
\end{aligned}
$$

The basic reproductive number $R_{0}=\rho\left(T V^{-1}\right)$ is simply calculated as the spectral radius of the matrix $T V^{-1}$.

\section{RESULTS}

Analysis of simulation data The infection curves predicted by our model (see Materials and Methods) are shown in Fig. 3. We note that our model predicts a fairly symmetric infection 
curve, whose peak trails behind the peak of the quarantined population. USA and India continue an upward growth, while Italy's infection curve has begun to drop. If current conditions continue without any new measures being taken, active cases are predicted to reach peak within a month for USA, and around five months for India. We also predict that everything will be normal in six months for Italy, without considering the effect of herd immunity. On the other hand, it may take a year at worst for India and USA to fully recover. By 'normal', we mean that the number of infected individuals have dropped below one thousand.

We observe a common trend in infected age-groups across all three countries, in that the young and middle aged groups (between 10 - 40 years of age) have the largest numbers of infected people, relative to the initial susceptible population size of that age-group. We estimate this by measuring the drop in susceptible population of age-group $i, S_{i}$, from it's initial value $S_{i, 0}$, and compare the values $\left(S_{i, 0}-S_{i}\right) / S_{i, 0}$ across all age-groups $i$ over time, as shown in Fig. 4 . This may be explained by larger contact coefficients $\alpha$ among themselves and other age-groups. The population within this age-group is also highly mobile, in each country. On the other hand, data suggests that infection is less common in children. A study in China (18) shows that out of 700 children who came in direct contact with the infected, only 10 caught the infection. Dr. Calum Semple, Professor at Liverpool University, stated that "We know that lung development doesn't finish until teenage years. ACE2 is highly regulated in lung development. Because of that the 'lock' might be expressed differently in kids' lungs" (19). Hence, we have identified the 15-20 year old age-group to be the most infected age-group across all three countries (Fig. 4). In our proposed control strategy, we thus place less emphasis on individuals younger than 15 years.

Considering different mortality rates across different age-groups has very little effect on the total infection and mortality curves, both qualitatively and quantitatively. We note that Italy's mortality curve in Fig. 2(F) has begun to flatten, far quicker than our model would suggest. We 
may explain this by noting that the reported mortality rates, which we used to fit our model, are likely inaccurate due to factors such as sampling bias and the changing capability of health care system. With time, as Italy continues to improve medical facilities and mobilize doctors and nurses, patients receive better care and facilities are no longer swamped as they were in the early stages of the pandemic. Presently, the overall mortality rate is highest in Italy, followed by India and USA (Fig. 2). Interpreting these mortality rates is complicated by the fact that pre-existing medical conditions play a major role, and are somewhat correlated with age. Fatality rates may also be inflated by limited testing, and the resulting selection bias in which asymptomatic individuals are not accounted for. In addition, the fact that our model omits 75-80 year agegroup in USA, despite a high expected mortality rate in that group, may explain why our model predicts a lower mortality rate in USA, compared to India and Italy.

The basic reproductive ratio $R_{0}$ can be interpreted as the expected number of cases directly caused by a single infected individual in a completely susceptible population. When $R_{0}>1$, the infection spreads in the population, and it does so more rapidly with higher $R_{0}$. When $R_{0}<1$, the infection eventually dies out, and the system proceeds towards the disease free equilibrium. We have calculated $R_{0}$ for the three countries, presented in Table $\mathrm{S} 1$. A lower $R_{0}$ value for India initially indicates a comparitively slower spread of disease than in USA and Italy. Fig. 5. illustrates the decrease of $R_{0}$ with the implementation of social distancing measures. The drop in $R_{0}$ is significant in all cases, although only Italy shows $R_{0}<1$, which is enough for the disease to die out.

Concerningly, our model shows very little impact of the lockdown in India, compared to the projections without one. After fitting our model to case numbers before the implementation of social distancing measures, our model predicts that complete stop of contact between people outside their home, or even a $50 \%$ reduction in such contact, is not consistent with subsequent reported cases. The three different countries show different degrees of reduction in contact, in 
terms of different control coefficients $\alpha$. This effective reduction in contact in India seems to be far less than in USA, which in turn is less than in Italy. In the case of Italy, data looks promising, suggesting that the implementation of lockdown was more successful.

New strategies and their impact The effectiveness of control strategies can be measured by the basic reproductive ratio $R_{0}$. The parameters which can practically reduce $R_{0}$ are the contact coefficients, $\alpha$ and the rate of quarantine of infected individuals, $\delta_{i}$. Social distancing works well at reducing interpersonal contact, but we can see that implementation issues can severely damage its effectiveness. By sufficiently increasing the number of tests carried out, we can identify and quarantine infected individuals more quickly, thus indirectly increaing $\delta_{i}$. This means that infected individuals would have a lower probability of infecting a susceptible individual. On the other hand, testing rates are limited by the medical resources of each country. While South Korea has managed to test a large fraction of their population, in countries with very large populations such as India, randomized testing for the entire population is not feasible. Hence, we suggest an age-group targeted testing initiative, where the age-grouops with the largest number of infected individuals is targeted. In addition, economic activities have come to a halt during lockdown in India. Barclays has estimated a loss of upto 234.4 billion USD in India (20). Practically, it may not be possible to continue a complete lockdown indefinitely. Only a multipronged approach can successfully combat an outbreak of infectious disease. Though we have not provided any medical insights, they should go hand in hand with the strategies we propose here.

We discuss the following two strategies below.

1. Keeping in mind that individuals in the age-group 15-40 years are most likely to catch infection, we emphasize testing more people from this age-group rather than randomized testing. This will help in isolating infected people and restrict their disease transmission. 
The impact of such a strategy is evident from the curves in Fig. 3(D). The corresponding change in $R_{0}$ is illustrated in Fig. 5(B). We see that although $R_{0}$ has not dropped below 1 , our strategy offers a significant improvement.

For this simulation, we have increased the value of $\delta_{i}$ for the targeted age-group 15-40 years, which corresponds to $i \in\{4,5,6,7,8\}$ (Table S2). The initial population sizes of these age-groups are shown in Table S3. We have assumed that infected members of these groups can proceed to quarantine in 4.8 days, on average. For the remaining groups, we increase the detection period to 5.4 days. Concentrating testing on groups most likely to have infected members helps bring them out of contact with the susceptible population, and hence flatten the infection curves. This in turn lowers the peak number of critical cases, thus distributing the workload of medical facilities over a longer period of time. The peak is also observed far later than with normal rates of testing, athough normalcy is restored not much later.

2. We suggest that a complete lockdown, followed by lockdowns in select regions is more effective than the reverse. As symptoms take time to manifest, infection can spread very rapidly to areas not under sufficient lockdown. However, if the majority of infected individuals can by identified and isolated by testing during the lockdown period, subsequent lockdowns can target those areas with larger infected populations. This would effectively reduce disease transmission across a country. We suggest that introducing a lockdown in slow phases, as was done in Italy and USA, may not have been as effective as a complete lockdown introduced in the early stages of the pandemic. However, we do acknowledge differences in the socioeconomic structures and dynamics of different countries, which demand differential strategies tailored individually to their underlying structures. Our model is ill equipped to model lockdowns in select regions, but we have approximated 
this effect with reduced contact coefficients $\alpha$.

\section{DISCUSSION}

Our model of COVID-19 dynamics allows us to make some useful predictions and modify preexisting strategies to obtain better results. This model has been tuned with available data of social contact matrices and reported deaths and infected individuals available till 30 April 2020. Methods of testing and social distancing are known to tackle this kind of situation. In the context of this novel disease we have re-examined these methods. It must be noted that in our model, we have interpreted the number of reported cases as the number of symptomatic or tested cases, and we assume that they are transferred to quarantine as soon as they are confirmed positive. Hence, our model has also predicted that the actual number of COVID-19 cases is much higher than reported.

We must note that as of 27 April 2020, India has conducted 5,00,000 tests. This is a major step up from the initial 15,000 tests before the declaration of lockdown. At this milestone of tests, India has recorded around 28,000 positive cases, as opposed to USA's 1,20,000 and Italy's 80,000 cases (21). India has also observed a comparitively lower mortality rate. This can be explained by her disproportionately young population, together with the low rate of infection and transmission in the younger age-groups. The mean age of India's population is 26.8, compared to USA's 38.2 and Italy's 45.4 (22).

We acknowledge that there are uncertainties in determining the model parameters due to unavailability of proper data, and that this may lead to incorrect predictions. The assumption that births, natural deaths, and aging are absent is valid only over relatively short periods of time. We have also focused on the population below 80 years of age, as the rest of the population is significantly small. With these assumptions we can clearly say that our model can make shortterm predictions, but cannot reliably make long-term forecasts. In this instance, we have run 
our simulation for a maximum time period of 10 months. We also acknowledge that partial lockdowns of infection hotspots are not well modeled by our method, which considers a given region as a whole. With the availability of reliable data, we may be able to appy our model on smaller populations and make region-wise predictions. We also postpone the application of targeted testing to other countries, such as USA and Italy, for further study.

Through this study, we have offered a more efficient, country-specific COVID-19 model for informing on strategies to contain the SARS-CoV-2 pandemic. Our contribution of a new age-stratified model will aid government and health agencies and will spur further research in COVD-19 modeling. The implications of our proposed work are timely as this is still an emerging situation in many countries and perhaps broad as well, as viral pandemics are predicted to keep re-emerging in near future, perhaps in different shades or shapes. Keeping in mind that all models are merely approximations of reality, we hope that this model can aid in developing policy, with economic and medical perspectives.

\section{References}

1. WHO, Coronavirus disease (COVID-2019) situation reports $-44,94$, https://www. who.int/emergencies/diseases/novel-coronavirus-2019/situatic

2. Worldometers.

https://www.worldometers.info/coronavirus/

3. P. Zhou, et al., A pneumonia outbreak associated with a new coronavirus of probable bat origin, Nature 579, 270-273 (2020).

4. D. Schenzle, An age-structured model of pre- and post-vaccination measles transmission, IMA Journal of Mathematics Applied in Medicine and Biology 1, 169-191 (1984). 
5. A. J. Kucharski, et al., The Contribution of Social Behaviour to the Transmission of Influenza A in a Human Population, PLOS Pathogens 10, 1-8 (2014).

6. X. Xiao, A. J. [van Hoek], M. G. Kenward, A. Melegaro, M. Jit, Clustering of contacts relevant to the spread of infectious disease, Epidemics 17, 1-9 (2016).

7. P. Rohani, X. Zhong, A. A. King, Contact network structure explains the changing epidemiology of pertussis, Science 330, 982-985 (2010).

8. J.S. Ayres, Surviving COVID-19, Sci. Adv. 6, eabc1518 (2020).

9. M. J. Keeling, P. Rohani, Modeling Infectious Diseases in Humans and Animals (Princeton University Press, 2011).

10. Wikipedia, https://en.wikipedia.org/wiki/2020_coronavirus_lockdown_in_India.

11. Axios, https://www.axios.com/italy-coronavirus-timeline-lockdown-deaths-cases-2

12. The New York Times, https://www.nytimes.com/article/coronavirus-timeline.html.

13. S. A. Lauer, et al., The Incubation Period of Coronavirus Disease 2019 (COVID-19) From Publicly Reported Confirmed Cases: Estimation and Application, Annals of Internal Medicine (2020).

14. WHO, Report of the WHO-China Joint Mission on Coronavirus Disease 2019, https://www.who.int/publications-detail/report-of-the-who-china-joint-mi 
15. PopulationPyramid,

https://www.populationpyramid.net.

16. K. Prem, A. R. Cook, M. Jit, Projecting social contact matrices in 152 countries using contact surveys and demographic data, PLOS Computational Biology 13, 1-21 (2017).

17. P. Van den Driessche, J. Watmough, Reproduction numbers and sub-threshold endemic equilibria for compartmental models of disease transmission, Mathematical Biosciences 180, 29-40 (2002).

18. Y. Xu, et al., Characteristics of pediatric SARS-CoV-2 infection and potential evidence for persistent fecal viral shedding, Nature Medicine 26, 502-505 (2020).

19. Financial Times, A. Ahuja, Scientists seek reason why coronavirus has less impact on children,

https://app.ft.com/cms/s/2d616ea0-7281-11ea-90ce-5fb6c07a27f2.html?sect i

20. The Hindu, https://www.thehindubusinessline.com/economy/barclays-slashes-india-grov

21. The Times of India, Ranchi Edition. 27 April, 2020.

22. Statista, https://www.statista.com.

\section{Acknowledgments}

Funding: This work was supported by Scientific \& Engineering Research Board (SERB), DST, Govt. of India under grant No. MTR/2017/000301 to the corresponding author (R.K. Upadhyay). 
Author contributions: R.K.U. and R.K.A. supervised the reasearch. R.K.U. and S.C. conceived and designed the approach. R.K.U., S.C. and S.S. developed the model. S.C. and S.S. implemented the model. R.K.U., S.C. and S.S. analysed and interpreted the data. R.K.U., S.C., S.S. and R.K.A. wrote the manuscript.

Competing interestS: The authors declare that they have no competing interests regarding the publication of this article.

Ethical standard: The authors state that this research complies with ethical standards. This research does not involve either human participants or animals.

Data and materials availibility: All data needed to evaluate the conclusions in the paper are present in the paper and/or the Supplementary Materials. Additional data related to this paper may be requested from the corresponding author.

\section{SUPPLEMENTARY MATERIALS}

Tables S1 to S4

Data files and Jupyter notebooks 


\section{Figures and Tables}

Fig. 1. The schematic diagram of the interacting population within a certain age class, for the model (2).

Fig. 2. The simulation data fitted to case data in (A) India, (B) USA and (C) Italy. Fatalities are shown in (D) India, (E) USA and (F) Italy. Note that the 'infected', 'quarantined', 'total infected', and 'dead' curves are simulated, while the 'actual cases' and 'actual deaths' datapoints have been obtained from Worldometers.

Fig. 3. The predicted variation in infected and quarantined populations in (A) India, (B) USA and (C) Italy. (D)The effect of targeted testing in India is shown by the dashed curves. The vertical green line marks the date 1 May 2020.

Fig. 4. The predicted relative decrease in susceptible populations by age-group in (A) India, (B) USA and (C) Italy.

Fig. 5. The variation of the basic reproductive number $R_{0}$ with time in (A) India, (B) India, with the effect of targeted testing in blue, (C) USA and (D) Italy.

Table S1. The basic reproductive number $R_{0}$.

\begin{tabular}{|l|ccc|}
\hline & India & USA & Italy \\
\hline$R_{0}(\mathrm{t}=0)$ & 1.78 & 1.87 & 2.03 \\
$R_{0}$ as of 30 April 2020 & 1.20 & 1.06 & 0.96 \\
$R_{0}$ with targeted testing & 1.16 & - & - \\
\hline
\end{tabular}

Table S2. The parameters of the model and their values.

\begin{tabular}{|c|l|l|}
\hline Parameter & Value & Source \\
\hline$\delta_{i}$ & $1 / 5.2$ & Referenced (13) \\
$\gamma$ & $1 / 14$ & Referenced (14) \\
$\epsilon$ & $1 / 15$ & Assumed \\
$\delta_{i}$ in targeted testing & $1 / 4.8$ when $4 \leq i \leq 8$ & Assumed \\
& $1 / 5.4$ when $i<4$ or $i>8$ & \\
\hline
\end{tabular}


Table S3. The initial susceptible population sizes by age class $i$. All population numbers are in millions.

\begin{tabular}{|l|l|rrr|}
\hline$i$ & Age class & India & USA & Italy \\
\hline 1 & $0-5$ & 116.78 & 19.60 & 2.38 \\
2 & $5-10$ & 119.95 & 20.29 & 2.72 \\
3 & $10-15$ & 126.98 & 21.15 & 2.87 \\
4 & $15-20$ & 125.69 & 21.25 & 2.87 \\
5 & $20-25$ & 121.80 & 22.44 & 2.96 \\
6 & $25-30$ & 116.68 & 23.74 & 3.18 \\
7 & $30-35$ & 111.06 & 22.70 & 3.38 \\
8 & $35-40$ & 101.34 & 21.28 & 3.71 \\
9 & $40-45$ & 88.16 & 20.15 & 4.32 \\
10 & $45-50$ & 77.72 & 20.14 & 4.90 \\
11 & $50-55$ & 67.43 & 20.87 & 4.90 \\
12 & $55-60$ & 57.95 & 21.69 & 4.56 \\
13 & $60-65$ & 47.72 & 20.43 & 3.86 \\
14 & $65-70$ & 36.58 & 17.42 & 3.53 \\
15 & $70-75$ & 22.84 & 13.76 & 3.25 \\
16 & $75-80$ & 14.66 & - & 2.61 \\
\hline & Total & 1353.34 & 306.90 & 56.08 \\
\hline
\end{tabular}

Table S4. The mortality rates $\mu_{i}$. 


\begin{tabular}{|l|ccc|}
\hline Age class & India & USA & Italy \\
\hline $0-5$ & 0 & 0 & 0 \\
$5-10$ & 0 & 0 & 0 \\
$10-15$ & 0.0009 & 0.0005 & 0 \\
$15-20$ & 0.0009 & 0.0005 & 0 \\
$20-25$ & 0.0009 & 0.0005 & 0.0004 \\
$25-30$ & 0.0009 & 0.0008 & 0.0004 \\
$30-35$ & 0.0014 & 0.0008 & 0.0007 \\
$35-40$ & 0.0016 & 0.0010 & 0.0014 \\
$40-45$ & 0.0023 & 0.0010 & 0.0021 \\
$45-50$ & 0.0023 & 0.0015 & 0.0028 \\
$50-55$ & 0.0054 & 0.0035 & 0.0053 \\
$55-60$ & 0.0081 & 0.0050 & 0.0081 \\
$60-65$ & 0.0153 & 0.0100 & 0.0186 \\
$65-70$ & 0.0252 & 0.0150 & 0.0294 \\
$70-75$ & 0.0315 & 0.0200 & 0.0543 \\
$75-80$ & 0.0540 & - & 0.0795 \\
\hline
\end{tabular}




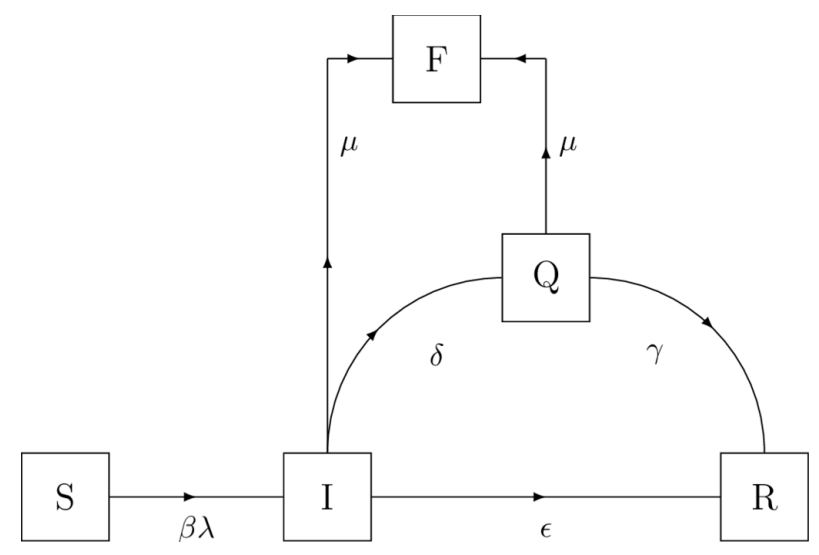

Figure 1:
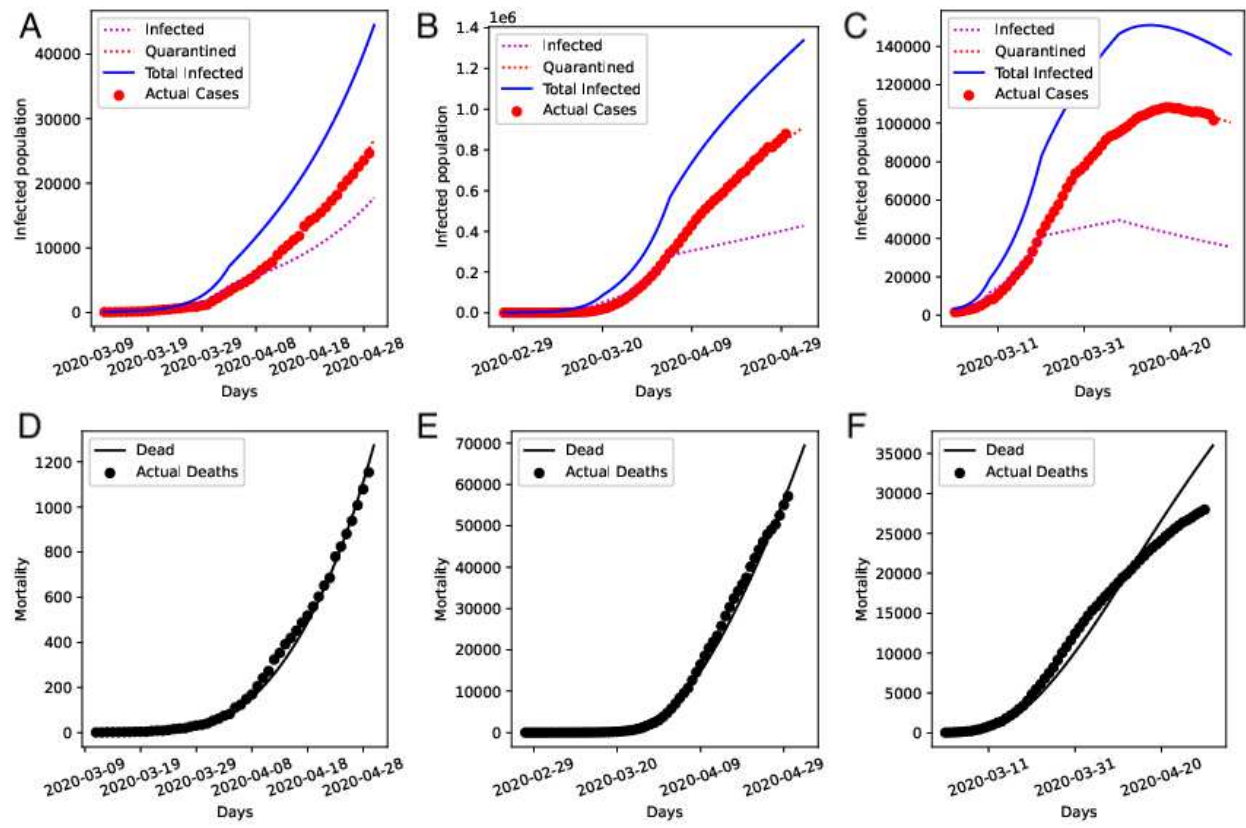

Figure 2: 

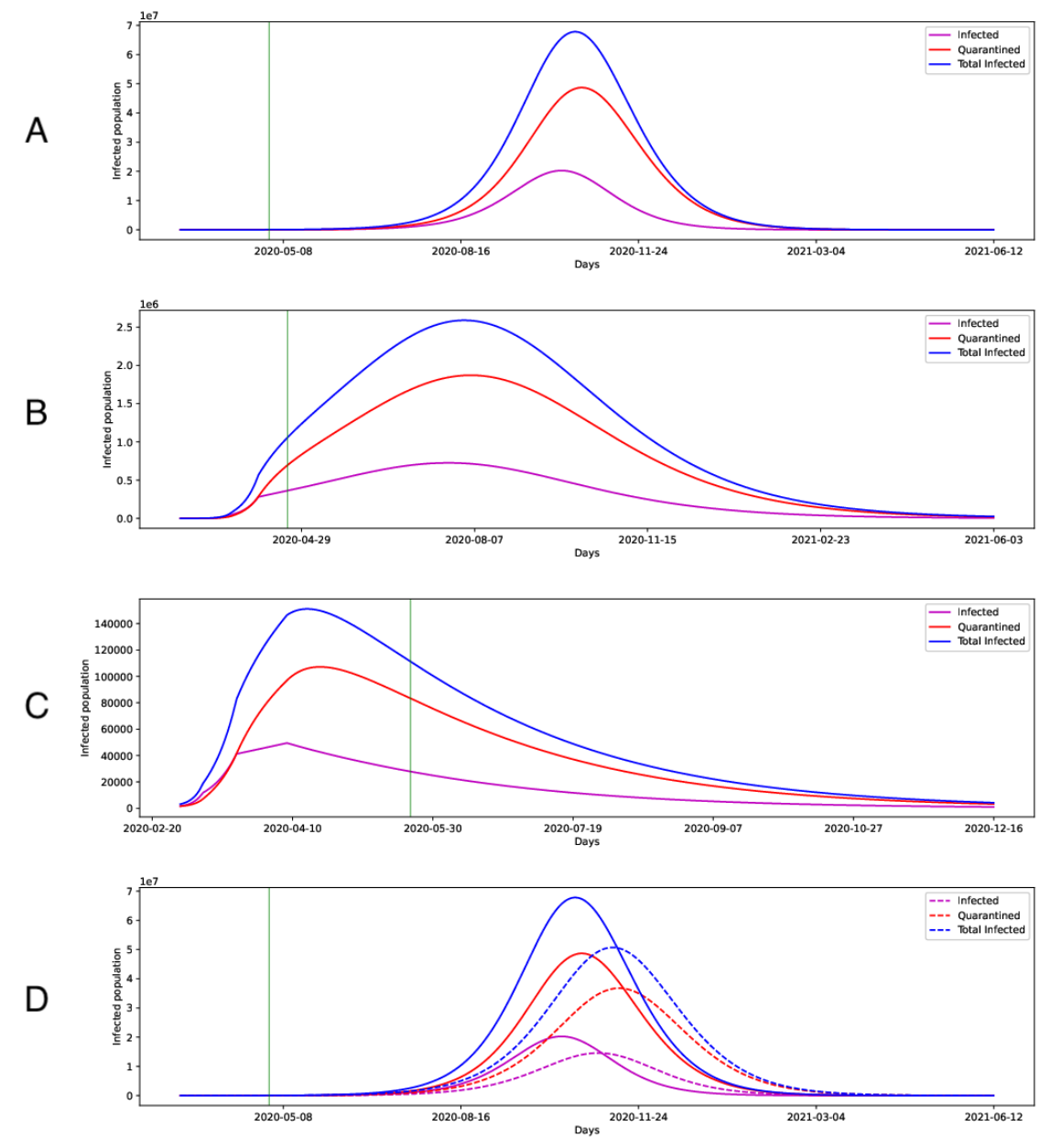

Figure 3: 

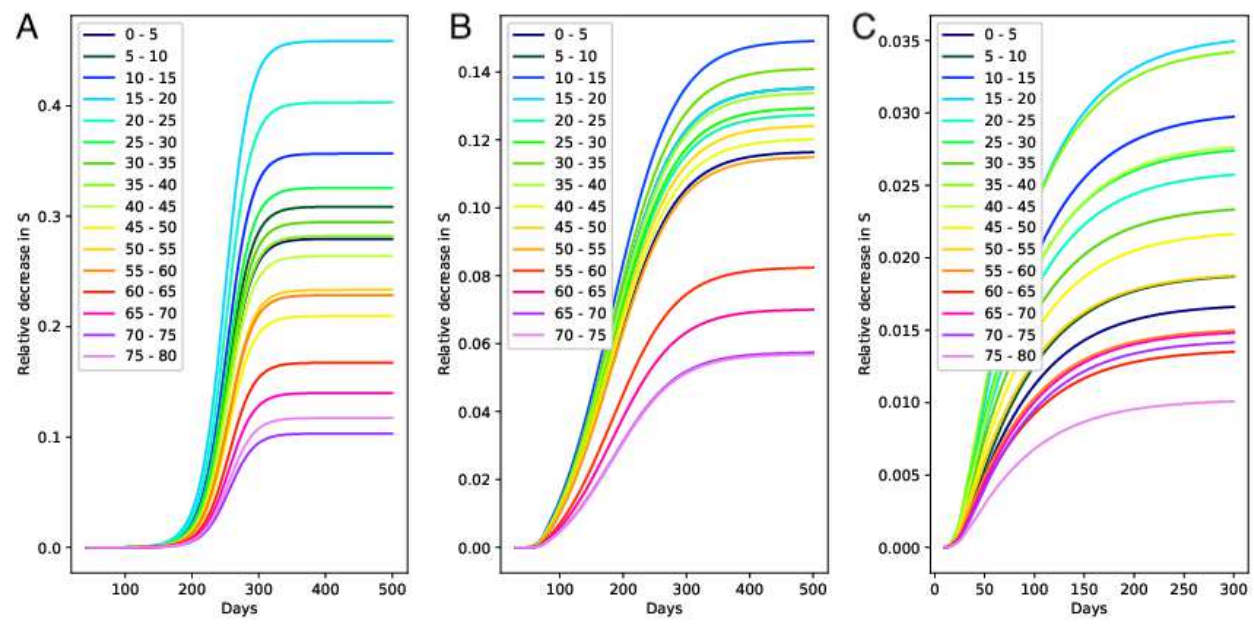

Figure 4:
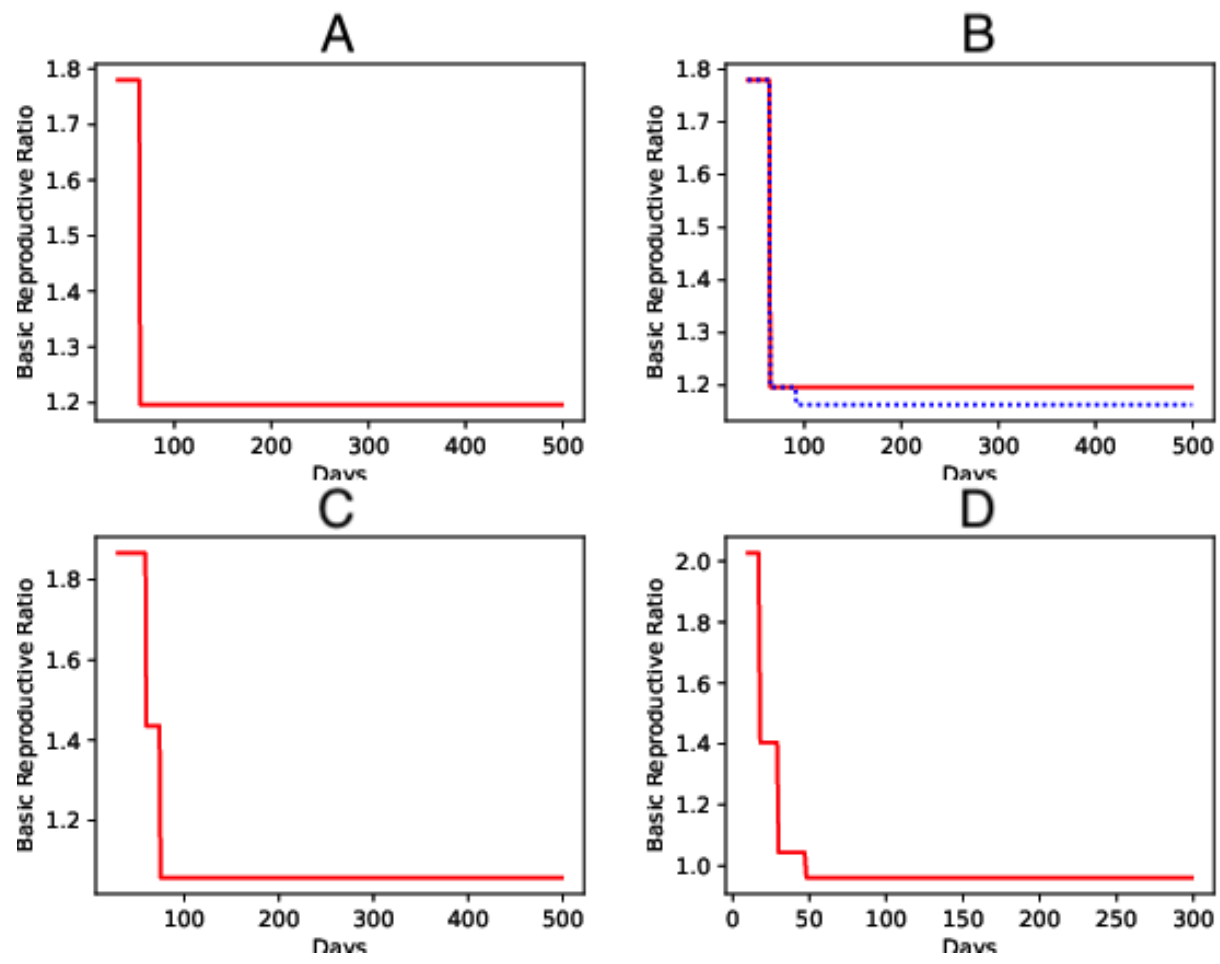

Figure 5: 
Figures

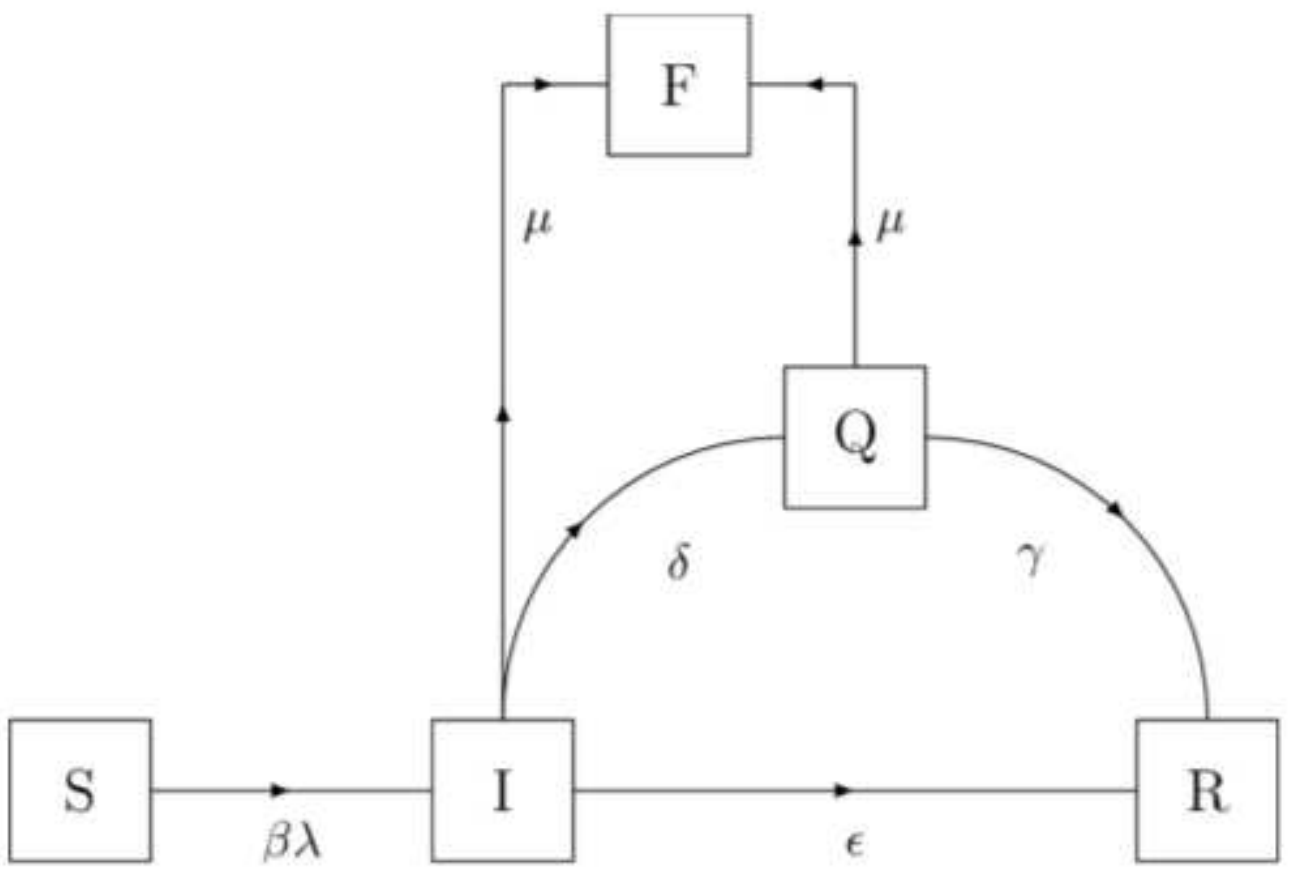

\section{Figure 1}

The schematic diagram of the interacting population within a certain age class, for the model (2). 

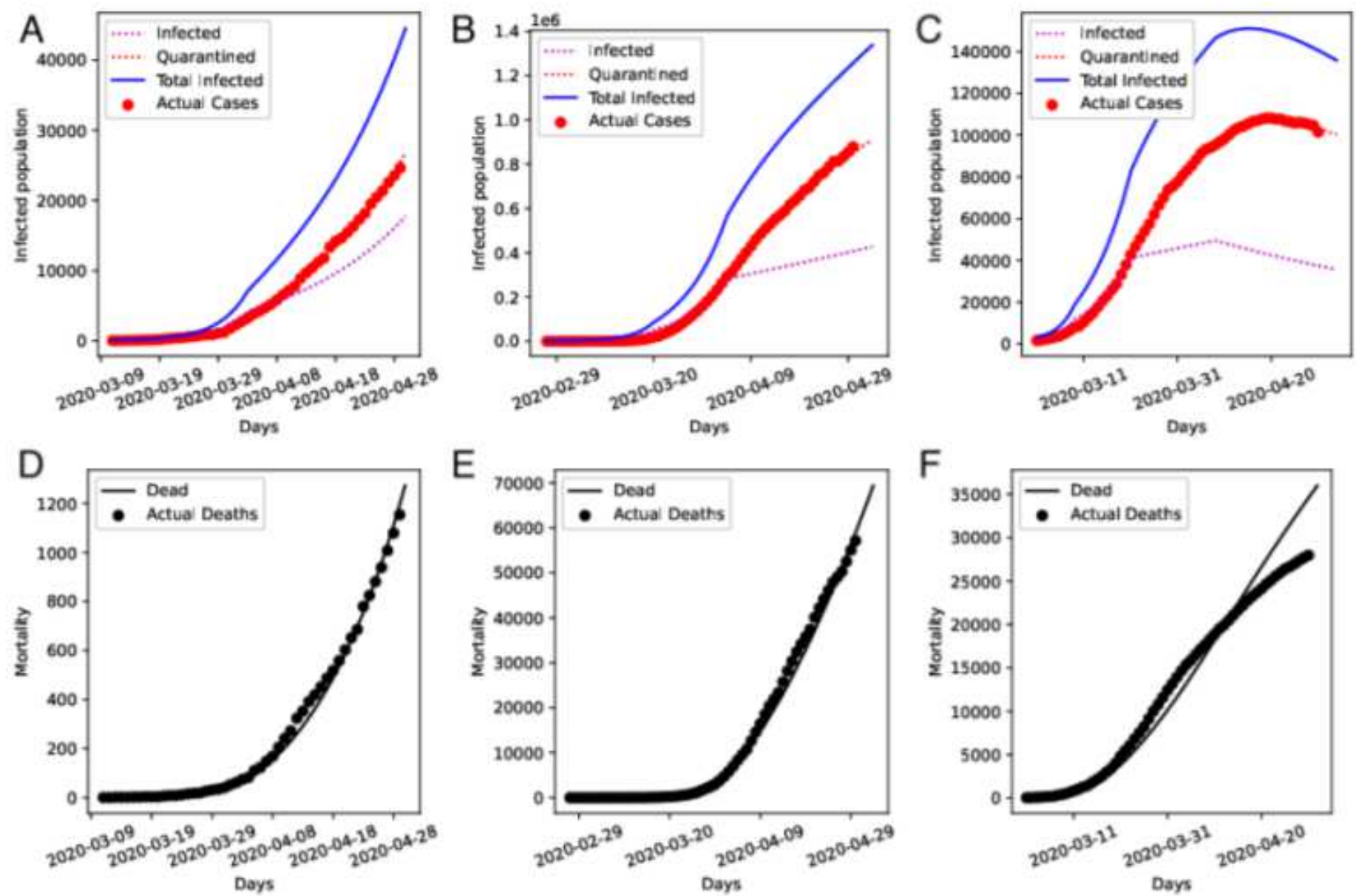

\section{Figure 2}

The simulation data fitted to case data in (A) India, (B) USA and (C) Italy. Fatalities are shown in (D) India, (E) USA and (F) Italy. Note that the 'infected', 'quarantined', 'total infected', and 'dead' curves are simulated, while the 'actual cases' and 'actual deaths' datapoints have been obtained from Worldometers. 

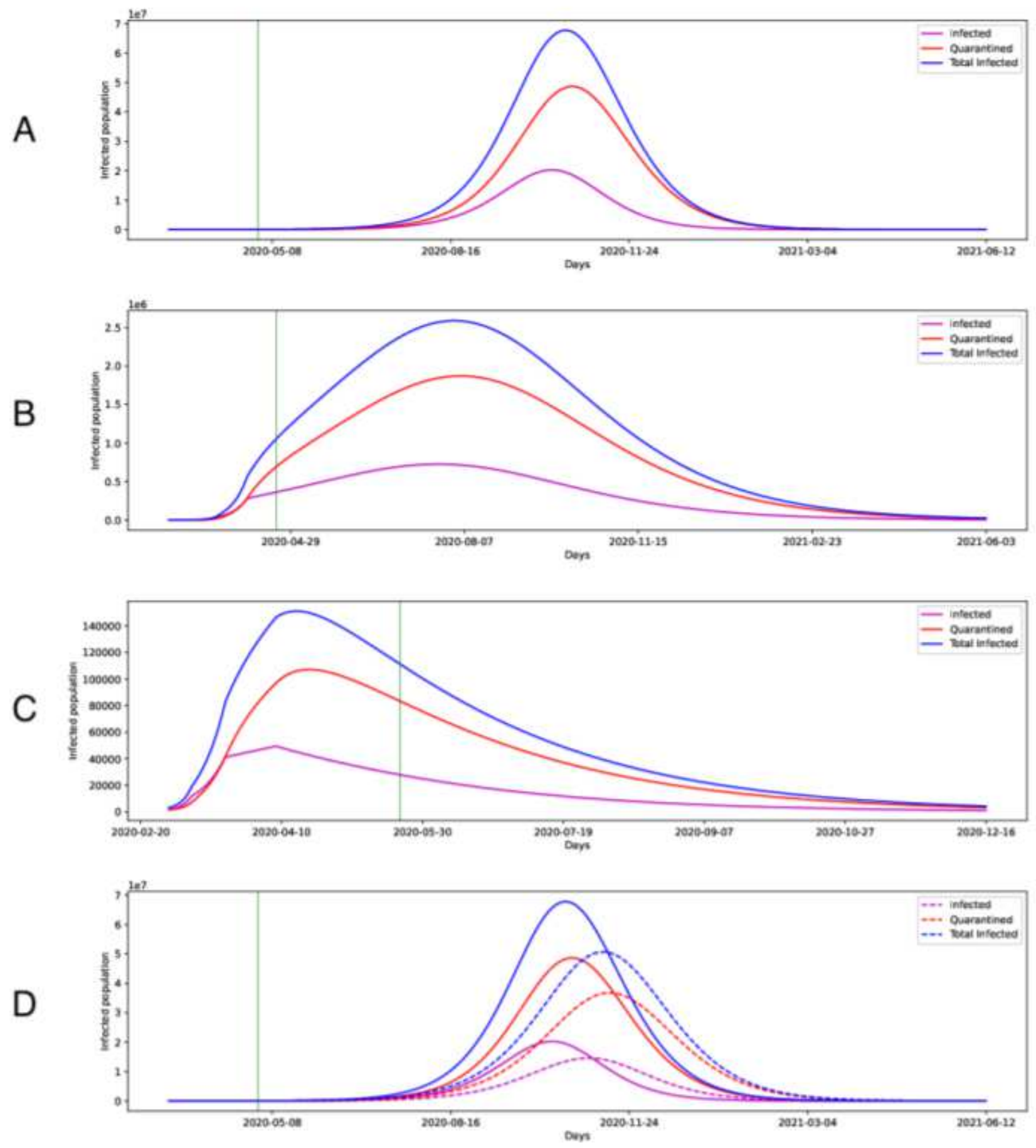

Figure 3

The predicted variation in infected and quarantined populations in (A) India, (B) USA and (C) Italy. (D)The effect of targeted testing in India is shown by the dashed curves. The vertical green line marks the date 1 May 2020. 

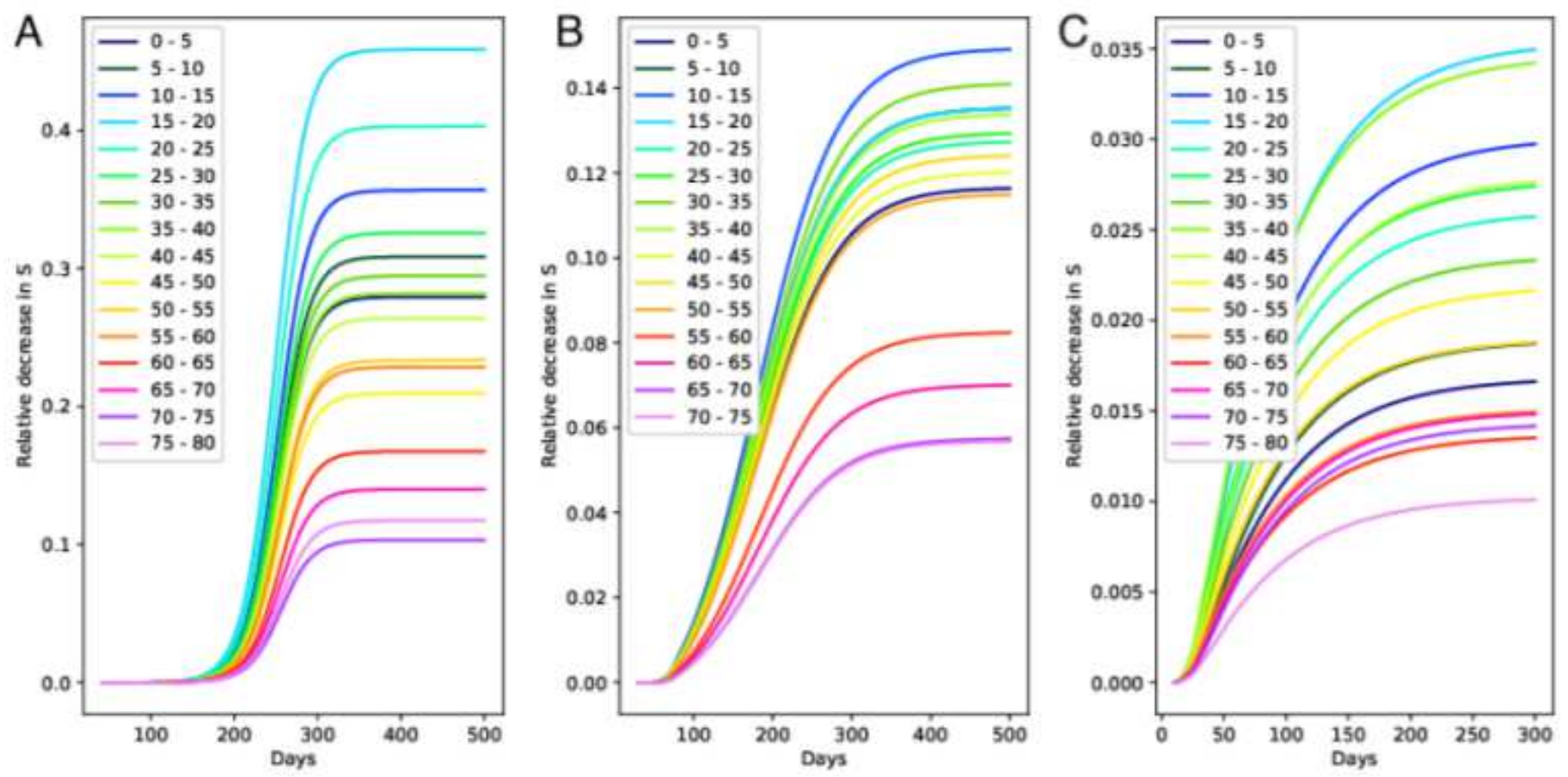

Figure 4

The predicted relative decrease in susceptible populations by age-group in (A) India, (B) USA and (C) Italy. 

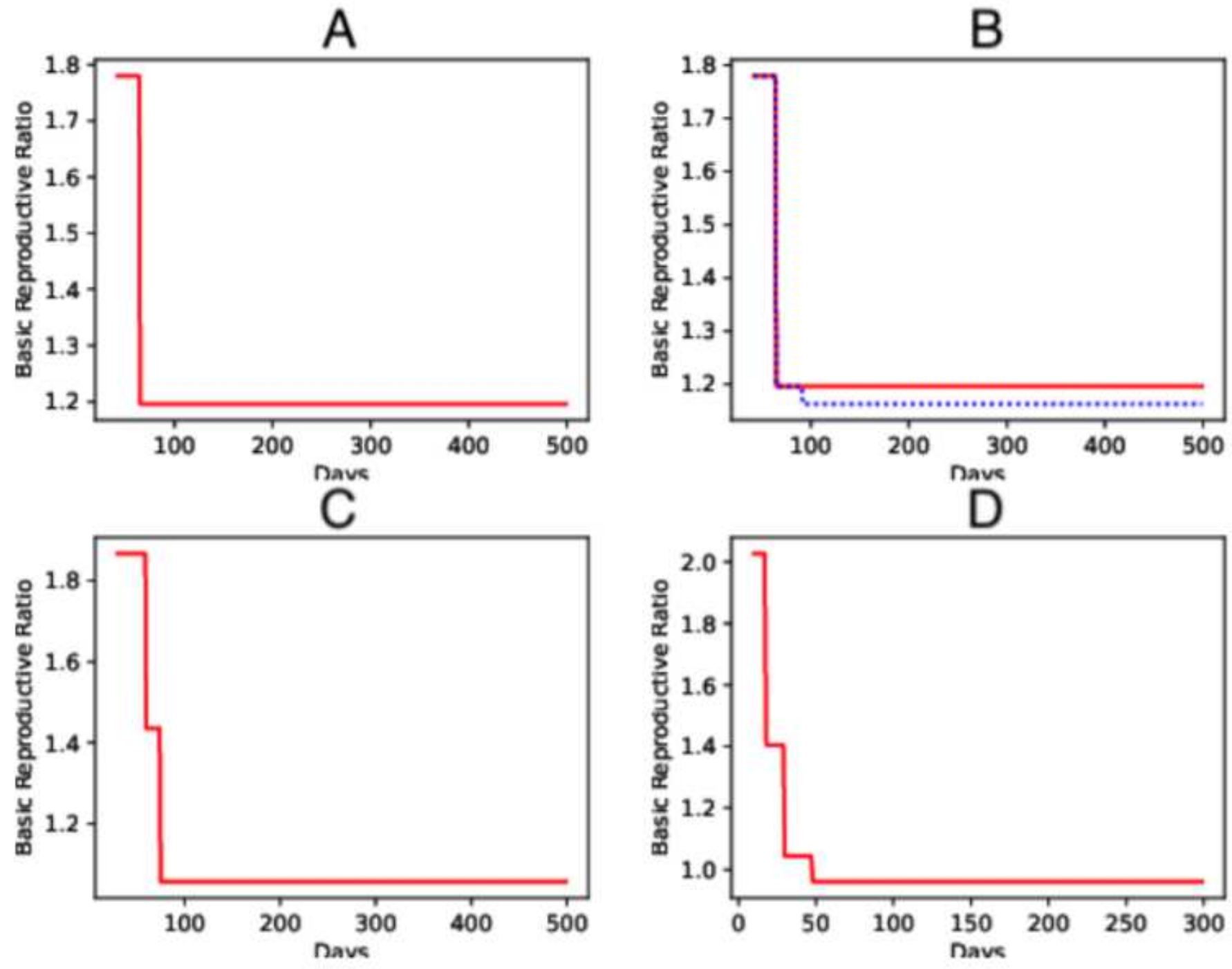

Figure 5

The variation of the basic reproductive number R0 with time in (A) India, (B) India, with the effect of targeted testing in blue, (C) USA and (D) Italy.

\section{Supplementary Files}

This is a list of supplementary files associated with this preprint. Click to download.

- data.zip 